\title{
Cognitive behavioural therapy significantly improves hypochondriacal symptoms
}

Barsky AJ, Ahern DK. Cognitive behavior therapy for hypochondriasis. A randomized controlled trial. JAMA 2004;291:1464-70.

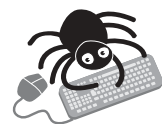

This article

contains extra text on the EBMH website

\section{Is cognitive behavioural therapy an effective treatment for hypochondriasis?}

METHODS

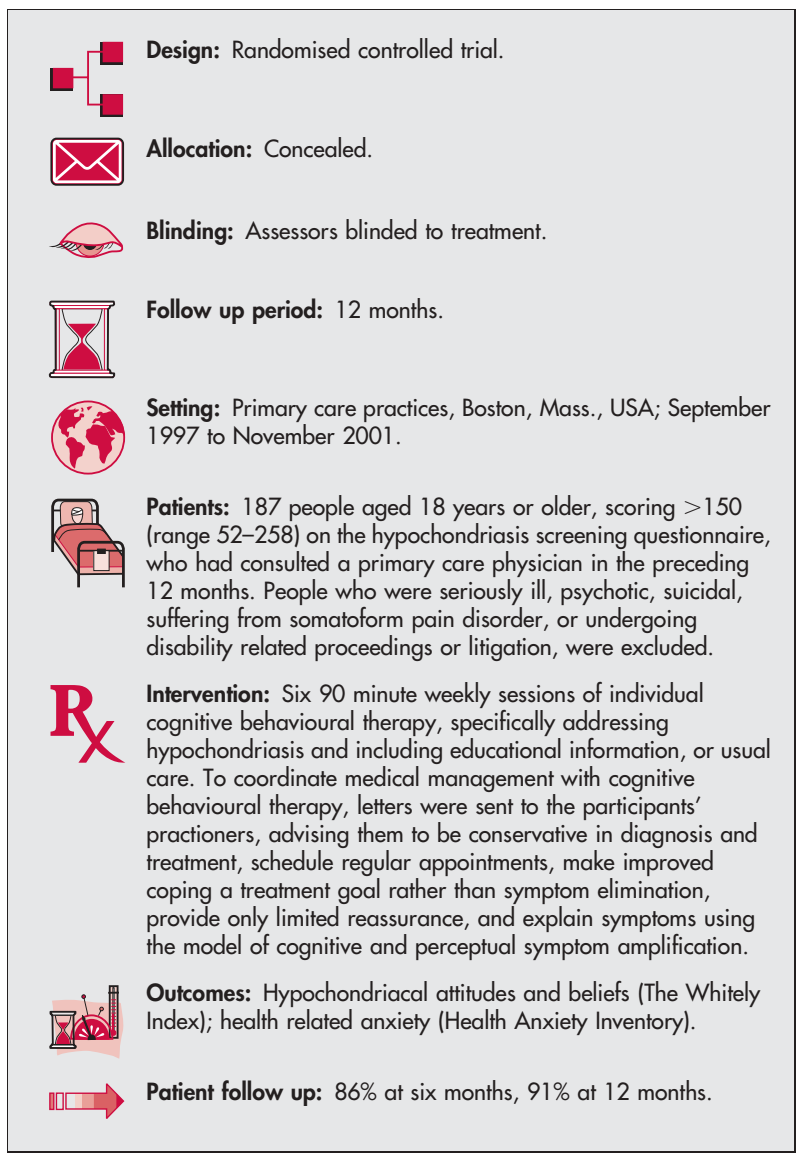

\section{MAIN RESULTS}

At 12 months, cognitive behavioural therapy significantly decreased hypochondriacal attitudes and beliefs $(\mathrm{p}<0.001)$ and health related anxiety $(\mathrm{p}<0.009)$ compared with usual care (see http://www. ebmentalhealth.com/supplemental for table). Ability to function socially was significantly improved $(\mathrm{p}<0.05)$ as was the ability to

For correspondence: Arthur J Barsky, Department of Psychiatry, Brigham and Women's Hospital, Boston, Massachusetts, USA; abarsky@partners.org Sources of funding: the National Institute of Mental Health. perform normal daily activities $(\mathrm{p}<0.001)$. There were no significant differences in improvement of somatic symptoms between groups.

\section{CONCLUSIONS}

Cognitive behaviour therapy significantly improves the symptoms of hypochondriasis and may have long term benefits for sufferers.

\section{Commentary}

7 his study claims to be the first to clearly look at the efficacy of CBT in a hypochondriacal patient population. Two groups participated: one which had six 90 minute sessions of CBT and the second group which received medical care as usual. In both groups, patients who fulfilled DSM IV criteria for hypochondriasis and those who only fulfilled subthreshold hypochondriasis were included. The primary care physicians of patients in the CBT group were asked to follow the following rules: (1) make improved coping with somatic symptoms rather than symptom elimination the goal of medical management; (2) schedule regular appointments; (3) provide only limited reassurance; (4) explain symptom amplification to the patient, and (5) be conservative in medical diagnosis and treatment. A total of 187 patients participated out of a total of 776 patients, of which 102 were in the CBT group and 85 in the medical as usual group. Not all patients completed the study. Of the 102 patients only $63(61.8 \%)$ attended all six sessions and of the 85,76 control patients ( $87 \%$ in person and $13 \%$ by telephone) completed the study. Results basically indicated that there was significant improvement in the treatment versus control group at both six and 12 months follow up. Hypochondriacal thoughts, health anxiety, and somatosensory amplification were all significantly improved via CBT. Only somatic symptoms were not significantly improved. This paper substantiates previous CBT studies in hypochondriasis ${ }^{1-3}$ that have shown CBT to be efficacious. Yaryura-Tobias \& Neziroglu ${ }^{4}$ have also indicated that attrition rate is greater with behavioural rather than cognitive or medical treatment as usual forms of treatment, as this study has found. Suggestions for primary care physicians are well formulated and should be followed. It is the opinion of the reviewer that more than six sessions of CBT are necessary to make long term gains in most hypochondriacal patients.

Fugen Neziroglu, PhD, ABBP Bio-Behavioral Institute, Great Neck, NY 11021 , USA

1 Warwick HMC, Clark DM, Cobb AM, et al. A controlled trial of cognitivebehavioral treatment of hypochondriasis. $\mathrm{Br} J$ Psychiatry 1996:169:189-95.

2 Neziroglu F, Anderson MC, Yaryura-Tobias JA. An in-depth review of obsessive-compulsive disorder, body dysmorphic disorder, hypochondriasis, and trichotillomania: therapeutic issues and curren research. Brief Treat Crisis Intervention 1999;5:59-94.

3 Salkovskis PM, Warwick MCH, Deale AC. Cognitive behavioral treatment for severe and persistent health anxiety. Brief Treat Crisis Intervention 2003;3:353-67.

4 Yaryura-Tobias JA, Neziroglu F. Obsessive compulsive disorder spectrum: pathogenesis, diagnosis and treatment. Washington DC: American Psychiatric Press, 1997. 